\title{
Spectroscopy and Control of Near-Surface Defects in Conductive Thin Film $\mathrm{ZnO}$
}

Leah L. Kelly, ${ }^{1}$ David A. Racke, ${ }^{1}$ Philip Schulz, ${ }^{2,3}$ Hong Li, ${ }^{4}$ Paul Winget, ${ }^{4}$ Hyungchul Kim, ${ }^{5}$ Paul Ndione, ${ }^{2}$ Ajaya K. Sigdel, ${ }^{2}$ Jean-Luc Brédas, ${ }^{6}$ Joseph J. Berry, ${ }^{2}$ Samuel Graham, ${ }^{5}$ and Oliver L. A. Monti ${ }^{1,(i) *}$

${ }^{1}$ University of Arizona, Department of Chemistry \& Biochemistry, 1306 E. University Blvd., Tucson, Arizona 85721, USA

${ }^{2}$ National Renewable Energy Laboratory, National Center for Photovoltaics, Golden, Colorado, 80401, USA

${ }^{3}$ Princeton University, Department of Electrical Engineering, Princeton, New Jersey 08544, USA

${ }^{4}$ Georgia Institute of Technology, School of Chemistry and Biochemistry and Center for Organic Photonics and Electronics, Atlanta, Georgia 30332-0400, USA

${ }^{5}$ Georgia Institute of Technology, School of Mechanical Engineering and Center for Organic Photonics and Electronics, Atlanta, Georgia 30332-0400, USA

${ }^{6}$ King Abdullah University of Science and Technology, Solar and Photovoltaics Engineering Research Center, Division of Physical Science and Engineering, Thuwal, 23955-6900, Saudi Arabia

(-) University of Arizona, Department of Physics, 1118 E. Fourth Street, Tucson, Arizona 85721, USA

e-mail address: monti@u.arizona.edu 


\section{RECEIVED DATE}

Tel: ++1 5206261177

Fax: ++1 5206218407 


\begin{abstract}
:
The electronic structure of inorganic semiconductor interfaces functionalized with extended $\pi$-conjugated organic molecules can be strongly influenced by localized gap states or point defects, often present at low concentrations and hard to identify spectroscopically. At the same time, in transparent conductive oxides such as $\mathrm{ZnO}$, the presence of these gap states conveys the desirable high conductivity necessary for function as electron-selective interlayer or electron collection electrode in organic optoelectronic devices. Here, we report on the direct spectroscopic detection of a donor state within the band gap of highly conductive zinc oxide by two-photon photoemission spectroscopy. We show that adsorption of the prototypical organic acceptor $\mathrm{C}_{60}$ quenches this state by ground-state charge transfer, with immediate consequences on the interfacial energy level alignment. Comparison with computational results suggests the identity of the gap state as a near-surface-confined oxygen vacancy.
\end{abstract}




\section{Introduction}

Conductive thin $\mathrm{ZnO}$ films have attracted significant attention in recent years for use in organic and hybrid organic-inorganic electronics because of their wide band gap $(\sim 3.4 \mathrm{eV})$, high electrical conductivity, earth abundance, and ease of fabrication processes compatible with organic semiconductor materials, including sol-gel, atomic layer deposition (ALD) and epitaxial growth. ${ }^{1-3}$ Inverted organic photovoltaic cells exploit $\mathrm{ZnO}$ and its inherent $n$-type conductivity in combination with the wide band gap to act as a hole-blocking / electron-selective layer between an organic active layer and a transparent electrode typically made from indium tin oxide (ITO). ${ }^{1-}$ ${ }^{3}$ This selectivity for charge carrier injection/extraction at the interface is critical for improved device functionality and efficiency of organic optoelectronics. ${ }^{1,4}$ Recent results indicate that the fundamental interactions that determine energy level alignment at the organic / inorganic interface are considerably different from the paradigmatic organic / metal interface. ${ }^{5-8}$ In particular, though defects and dopants are generally thought to convey the $n$-type conductivity observed in $\mathrm{ZnO}$, their precise role and nature is at present still controversial and unclear. ${ }^{9-12}$

$\mathrm{ZnO}$ native point defects have been extensively studied as a means of understanding the intrinsic $n$-type nature of bulk $\mathrm{ZnO}$. Photoluminescence (PL), ${ }^{9,13}$ electron paramagnetic resonance (EPR), ${ }^{13-16}$ and deep level transient spectroscopy ${ }^{17-20}$ have all been used to uncover the nature and electronic structure of the various point defects. Three species in particular have been widely discussed as candidates for the pervasive $n$-type nature of $\mathrm{ZnO}$ : oxygen vacancies $\left(\mathrm{O}_{\mathrm{v}}\right)$, zinc interstitials $\left(\mathrm{Zn}_{\mathrm{i}}\right)$, and atomic hydrogen. ${ }^{9,11,21-23}$

Much less is known however regarding the electronic structure and role of point defects and dopants in the near-surface region of $\mathrm{ZnO}$. Only a few studies have investigated the role of 
surface defects on the electronic structure, and an understanding of the impact of surface and near-surface defects on hybrid organic / inorganic semiconductor interfacial electronic structure and charge transfer is only starting to emerge. ${ }^{5,10,24-28}$ Most of these studies have relied on indirect methods for experimental detection of near-surface gap states, somewhat impeding a full understanding of the importance of gap states on the interfacial electronic structure of highly conductive $\mathrm{ZnO}$ surfaces functionalized by organic semiconductor adsorbates. ${ }^{5,24,26}$

Here we show for the first time that two-photon photoemission (2PPE) spectroscopy can be used on highly conductive, polycrystalline thin $\mathrm{ZnO}$ films fabricated by plasma-enhanced atomic layer deposition (peALD) to directly reveal a density of states (DOS) located within the $\mathrm{ZnO}$ band gap. 2PPE accesses both ground and excited states, and is readily tunable over a range of photon energies that span the band gap. By careful choice of excitation energies that make photoemission from the valence band maximum impossible, our measurements select uniquely for states within the $\mathrm{ZnO}$ bandgap and situated in the near-surface region.

Our study allows a comparison to current models of the surface electronic structure in highly conductive $\mathrm{ZnO}$, both bare and functionalized by the prototypical organic acceptor $\mathrm{C}_{60}$, suggesting for the first time an assignment of the microscopic origin of the gap state DOS. ${ }^{10}$ The paper is organized as follows: We first present the characterization of the valence band of high conductivity $\mathrm{ZnO}$ thin-films by ultraviolet photoemission spectroscopy (UPS), followed by an investigation of the gap state DOS with 2PPE. We next show that this DOS is altered considerably by functionalization with $\mathrm{C}_{60}$ or by mild annealing. Finally, we assign the origin and nature of defect states by comparison with state-of-the-art computational results. The implications of our findings extend likely beyond $\mathrm{ZnO}$ to other electronically active surfaces such as other oxides or perovskites. 


\section{Experimental}

\section{(a) ZnO preparation}

A detailed description for the preparation of plasma-enhanced atomic layer deposited (peALD) ZnO thin films has been given previously. ${ }^{24}$ Briefly, indium zinc oxide (IZO) films were sputter-deposited on glass substrates to provide a planar substrate for $\mathrm{ZnO}$ film growth. $\mathrm{ZnO}$ was then grown by peALD, at $100^{\circ} \mathrm{C}$ and 0.4 torr, pulsing diethyl zinc as a precursor into the chamber, followed by exposure to a remote oxygen plasma using a $30 \mathrm{sccm}$ oxygen flow rate at $300 \mathrm{~W}$ RF power for 20 seconds. This process was cycled 200 times for the $\mathrm{ZnO}$ samples, yielding approximately $20 \mathrm{~nm}$ thick films as measured using spectroscopic ellipsometry on a $\mathrm{Si}$ witness substrate, present during the thin film growth process (M-2000, J. A. Woollam Co.). The peALD-ZnO films grown in this fashion were polycrystalline and preferentially oriented towards both the non-polar (100) and the polar (002) face as determined by X-ray diffraction. ${ }^{24}$

\section{(b) $\mathrm{C}_{60}$ deposition}

$\mathrm{C}_{60}(99 \%$ pure) was purchased from MER Corp., triply sublimated using a home-built resublimation apparatus, and deposited using a home-built Knudsen cell. Film thickness was determined using a previously calibrated quartz crystal microbalance (QCM). $\mathrm{C}_{60}$ thin films were grown at a rate of $0.5 \AA / \mathrm{min}$ in a deposition chamber at a base pressure of $4 \times 10^{-9} \mathrm{mbar}$. As film formation is not expected to strictly follow layer-by-layer growth, we report film thickness in terms of monolayer equivalents (MLE), where 1 MLE corresponds to $8 \AA$.

\section{(c) Photoelectron spectroscopy}

The sample was introduced into the photoelectron spectrometer (VG EscaLab MK II, base pressure of $3 \times 10^{-10}$ mbar) equipped with an Ar ion sputter gun ( $5 \mu \mathrm{A}$ sample current, $0.7 \mathrm{kV}$ 
acceleration voltage) and an integrated sample heater. When annealing, the $\mathrm{ZnO} / \mathrm{IZO} /$ glass sample was kept at or below $130^{\circ} \mathrm{C}$, measured with a pyrometer (Raytek, $30 \mathrm{~cm}$ from sample), in order to prevent migration alloying of the $\mathrm{IZO}$ substrate with the $\mathrm{ZnO}$ films. The electronic structure was analyzed first by ultraviolet photoemission spectroscopy (UPS) to confirm workfunction and surface electronic structure. ${ }^{29}$ The measured workfunction of the $\mathrm{ZnO}$ films prepared in this manner was 3.7(1) eV, consistent with previous work and the existence of shallow donor gap states. ${ }^{5,10,24}$ Surfaces prepared in this manner were stable over extended periods (14 days) of exposure to both $\mathrm{He}(\mathrm{I})$ and (II) radiation and the ultrahigh vacuum conditions in the spectrometer chamber, indicating the absence of hydrogen adlayers metalizing

the surface, $\mathrm{H}_{2} \mathrm{O}$ adsorption and photoinduced desorption of weakly bound adsorbates. ${ }^{8,30,31} \mathrm{We}$ verified the absence of alloying with IZO by x-ray photoemission spectroscopy (XPS), taken at normal incidence and grazing angle in a Kratos Axis Ultra spectrometer using Al Ka radiation (1486.7 eV). No band bending was observed by XPS, consistent with the high carrier concentrations typical for these films. ${ }^{24}$

\section{(d) Two-photon photoemission}

2PPE was performed using the frequency-doubled output of a $15 \mathrm{~W}$ pumped Ti:Sapphire oscillator (80 MHz repetition rate, 90 fs pulse duration, fundamental photon energies 1.2 to 1.7 $\mathrm{eV}$ ). Following dispersion-compensation in a prism pair, the pulse train was introduced into the UHV chamber through a fused silica viewport, incident on the sample in p-polarization and at an angle of $53^{\circ}$ with respect to the surface normal. Pulse energy, typically around $250 \mathrm{pJ} /$ pulse, was set by a neutral density filter wheel. When carrying out 2PPE measurements on semiconductor surfaces, it is possible for surface photovoltage (SPV) effects to shift the spectra. Here, no SPV could be detected in either XPS, UPS or 2PPE over a range of excitation densities, 
consistent with the highly conductive and near-degenerately doped nature of the thin $\mathrm{ZnO}$ films. ${ }^{24,25}$ No evidence of surface photovoltage effects or radiation damage was observed across the range of excitation intensities used $(250-500 \mathrm{pJ} /$ pulse $)$.

All 2PPE spectra were collected at room temperature, and the photoelectron kinetic energies were referenced to the Fermi level $E_{\mathrm{F}}$. For the spectra shown here, the take-off angle was normal to the surface, and $-3 \mathrm{~V}$ sample bias and $5 \mathrm{eV}$ pass energy were used. The polycrystalline nature of the $\mathrm{ZnO}$ films inherently results in the measurement of electronic structure averaged over a wide range of possible electron momenta.

All 2PPE spectra are presented in terms of final state energy, $E_{f i n a l}$, as shown in Figure 1. This energy is the energy above the Fermi level after absorption of two photons. It depends both on the photon energy and the initial occupation of the probed state. In the event that there is no dispersion in $k_{\perp}, E_{\text {final }}$ is defined as $\left(E-E_{\mathrm{F}}\right)+\Delta$, where $E$ is the energy of the probed state relative to $E_{\mathrm{F}}$, and $\Delta=2 h v$ for occupied levels - ionized by coherent absorption of two photons -, while $\Delta=h v$ for unoccupied levels, excited by a first photon and subsequently ionized by a second photon. A plot of the peak center $\left(E_{\text {final }}\right) v s$. photon energy $(h v)$ determines whether an observed feature corresponds to an occupied or an unoccupied level. Since the $\mathrm{ZnO}$ films used in these experiments are polycrystalline, the use of this analysis is appropriate.

Figure 1: Energy level diagram of $\mathrm{ZnO}$ including shallow defects, and schematic representation of the $2 \mathrm{PPE}$ process. $E_{F}, \mathrm{~V}_{\mathrm{L}}, \Phi, \mathrm{CBM}, \mathrm{VBM}$ and DS denote Fermi level, vacuum level, workfunction, conduction band minimum, valence band maximum, and defect state, respectively. The left excitation process shows that direct photoemission by coherent absorption of two photons from the VBM is energetically not allowed for the chosen photon energies. 


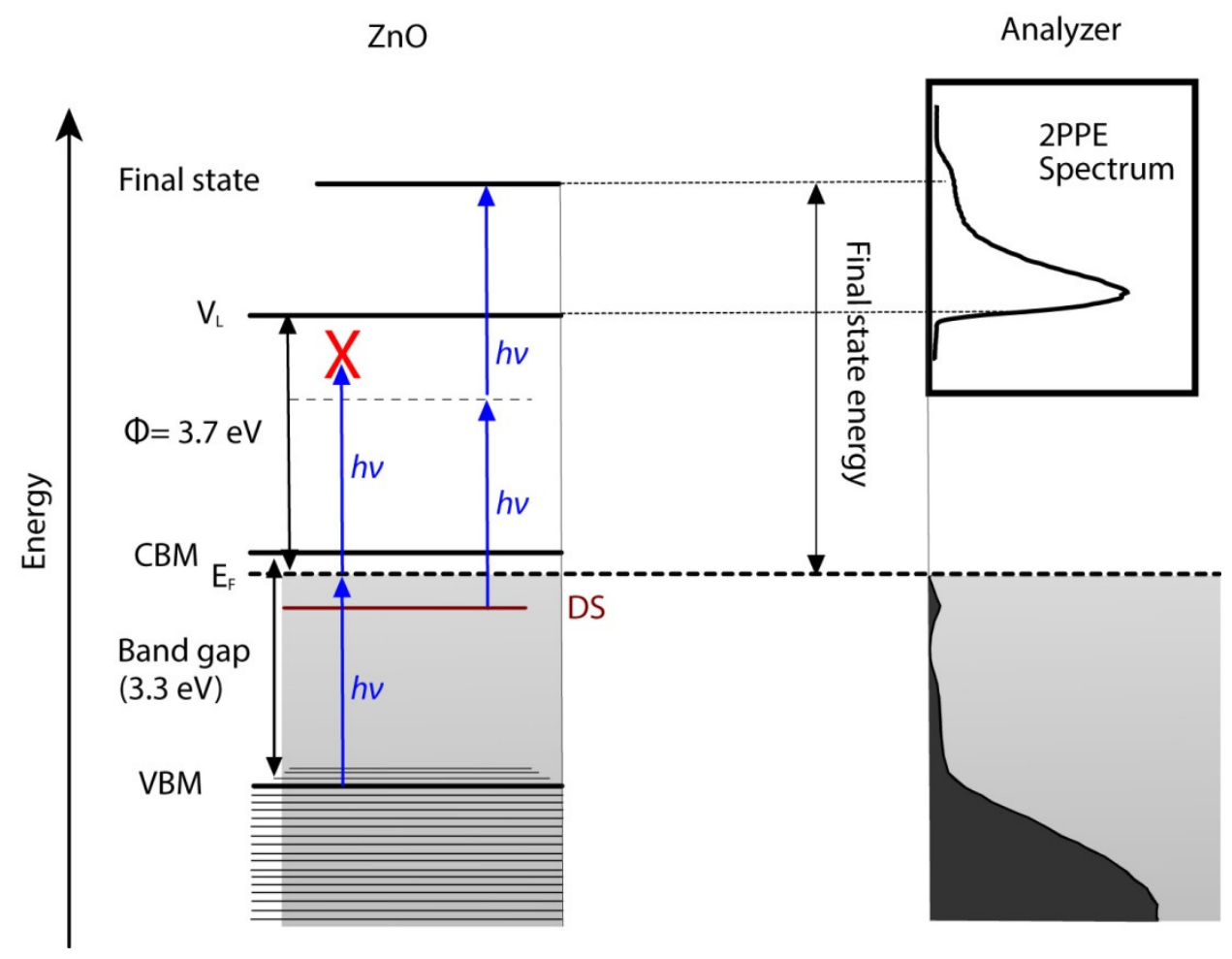

\section{III.Results and Discussion}

\section{(a) Valence Band Structure}

We first investigate the near-surface electronic structure of the $n$-ZnO films by UPS (Figure 2). The workfunction $\Phi=3.7(1) \mathrm{eV}$ and valence band onset of 3.5(1) $\mathrm{eV}$ with respect to $E_{\mathrm{F}}$ are consistent with previous work on similar thin $\mathrm{ZnO}$ films and are in good agreement with the strongly $n$-type nature of the films (carrier concentration of $10^{19} \mathrm{~cm}^{-3}$ ). ${ }^{5,10,24,25}$ Given a bandgap of $E_{g}=3.4 \mathrm{eV}$, the valence band onset suggests that these films are close to or in the degenerate doping regime. In addition to the spectroscopic features typically observed for UPS of $\mathrm{ZnO}$, we also find a high density of tail states extending past the valence band onset deep into the bandgap (Figure 2c). The tail states reside on a background created by satellite emission from $\mathrm{HeI} \beta$, as indicated in the figure by the shifted and rescaled spectrum $(1.87 \mathrm{eV}$ and to $2 \%$ of the HeI $\alpha$ signal, respectively). This density of tail states is typically associated with deep lying 
defect levels such as $\mathrm{Zn}_{\mathrm{v}}$ and bulk $\mathrm{O}_{\mathrm{v}}$. Together with shallow donor defects it confers the low workfunction of 3.7(1) to these films, as discussed in several recent combined computational / spectroscopic studies. ${ }^{5,10}$ In contrast, pristine single crystalline $\mathrm{ZnO}$ surfaces have workfunctions around $4.5 \mathrm{eV}$ and do not show significant band tailing into the gap. ${ }^{29}$ The close-up view of the gap region (Figure $2 \mathrm{c}$ ) also shows that no density of states can be detected within $\sim 1 \mathrm{eV}$ of $E_{\mathrm{F}}$ in UPS, despite the fact that shallow donors must be present to account for the $n$-type character of $\mathrm{ZnO} .^{9,23}$ This spectroscopically dark band gap is consistent across a range of highly conductive $\mathrm{ZnO}$ films prepared in a wide variety of ways and oriented towards different crystal faces, as reported previously. $5,10,24$

Figure 2: UV photoemission spectra (UPS) of peALD ZnO showing (a) secondary electron cutoff, (b) valence band region and onset (blue dashed line), and (c) close-up of valence band maximum and bandgap region.
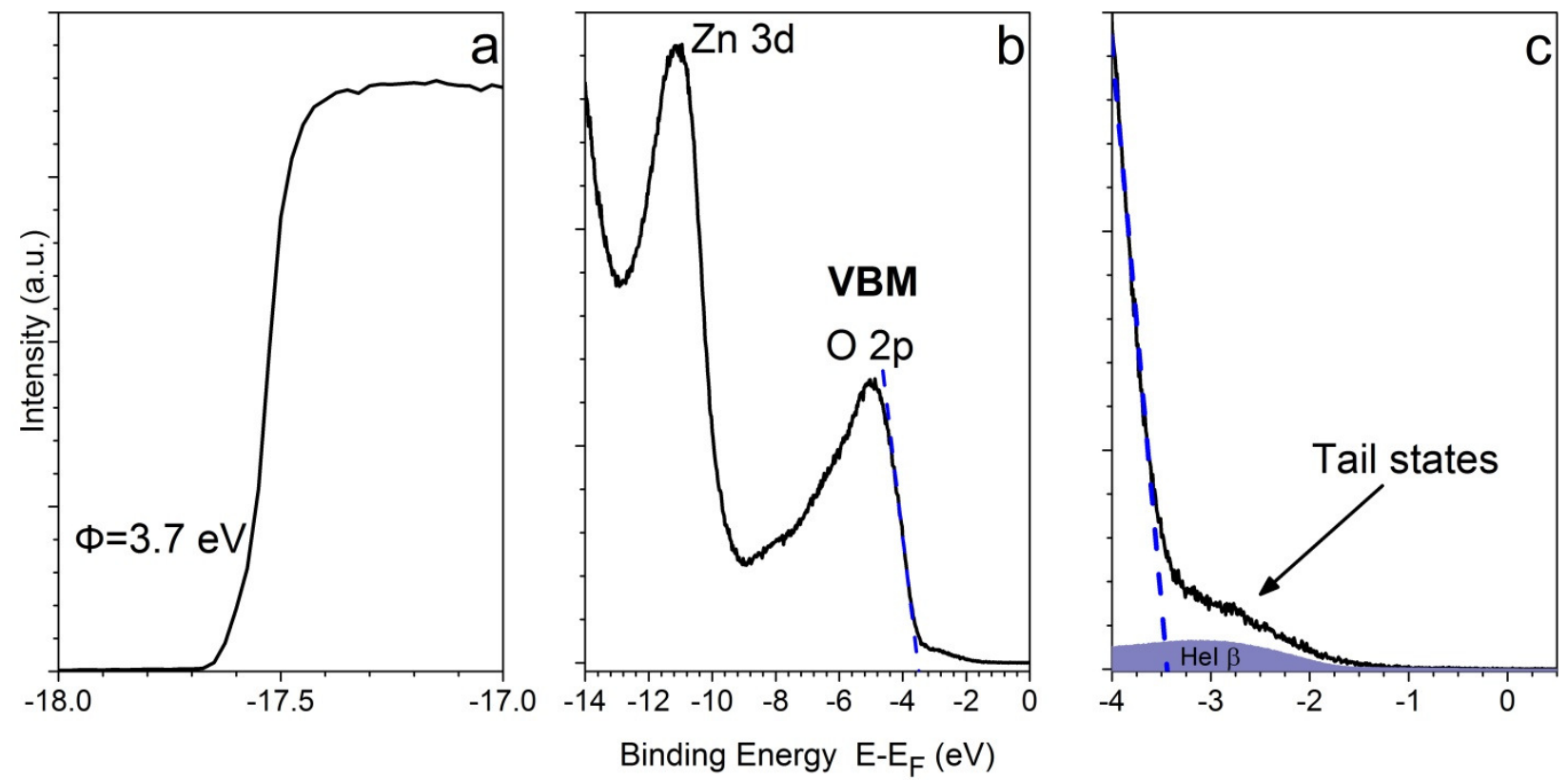


\section{(b) Spectroscopy Inside the Gap}

Due to the easily varied and low photon energy, $2 \mathrm{PPE}$ is ideally suited to probe the band gap region in more detail, taking advantage also of potentially different photoemission cross-sections in this energy range. In order to restrict spectroscopic detection to states inside the bandgap, either from the tail extending above the VBM or from a density of states closer to $E_{F}$, we used a range of photon energies $(2 \omega, 2.76-3.35 \mathrm{eV})$ below the band gap energy. Since the $\mathrm{ZnO}$ ionization energy for our $\mathrm{ZnO}$ films is $>7 \mathrm{eV}$, these low photon energies prevent two-photon photoemission from valence band states. We exclude three-photon photoemission (3PPE) due to insufficiently high excitation power densities, as considerably larger pulse fluences would be needed for 3PPE to occur. ${ }^{32,33}$ Since for the chosen range of photon energies all levels available to electronic excitation from the valence band are below $E_{F}$ and thus already occupied, resonant 3PPE processes are excluded as well. The experimental conditions prevent thus photoemission from occupied valence band states.

An overview 2PPE spectrum taken with a photon energy of $3.04 \mathrm{eV}$ is shown in Figure 3a. All 2PPE spectra are dominated by the scattered secondary electron background at the lowest final state energies, as is typical for $2 \mathrm{PPE}$ spectra of oxides. ${ }^{34,35}$ Beyond these slow electrons, a feature at the highest final state energy, approximately $5.70 \mathrm{eV}$ and labeled DS (defect state) in Figures $3 \mathrm{a}$ and $3 \mathrm{~b}$, is reproducibly observed at all photon energies used. A close-up of this region of the 2PPE spectrum is shown in Fig. 3b, showing a distinct spectral feature emerging as a shoulder between final state energies of $5.3 \mathrm{eV}$ and $5.9 \mathrm{eV}$, and the corresponding fit with a Gaussian envelope is shown as well. A linear background was also fit to adequately match the tail of the secondary electron background. Figures $3 a$ and $b$ show the spectra also in terms of an initial state / binding energy scale, revealing that DS is located within a few hundred meV of $E_{F}$. 
Figure 3: (a) Full 2PPE spectrum taken with a photon energy of $3.04 \mathrm{eV}$. (b) Close up of region near $E_{\mathrm{F}}$. (c) Stacked 2PPE spectra for different photon energies. (d) Photon energy dependence plot (final state energy vs. photon energy).
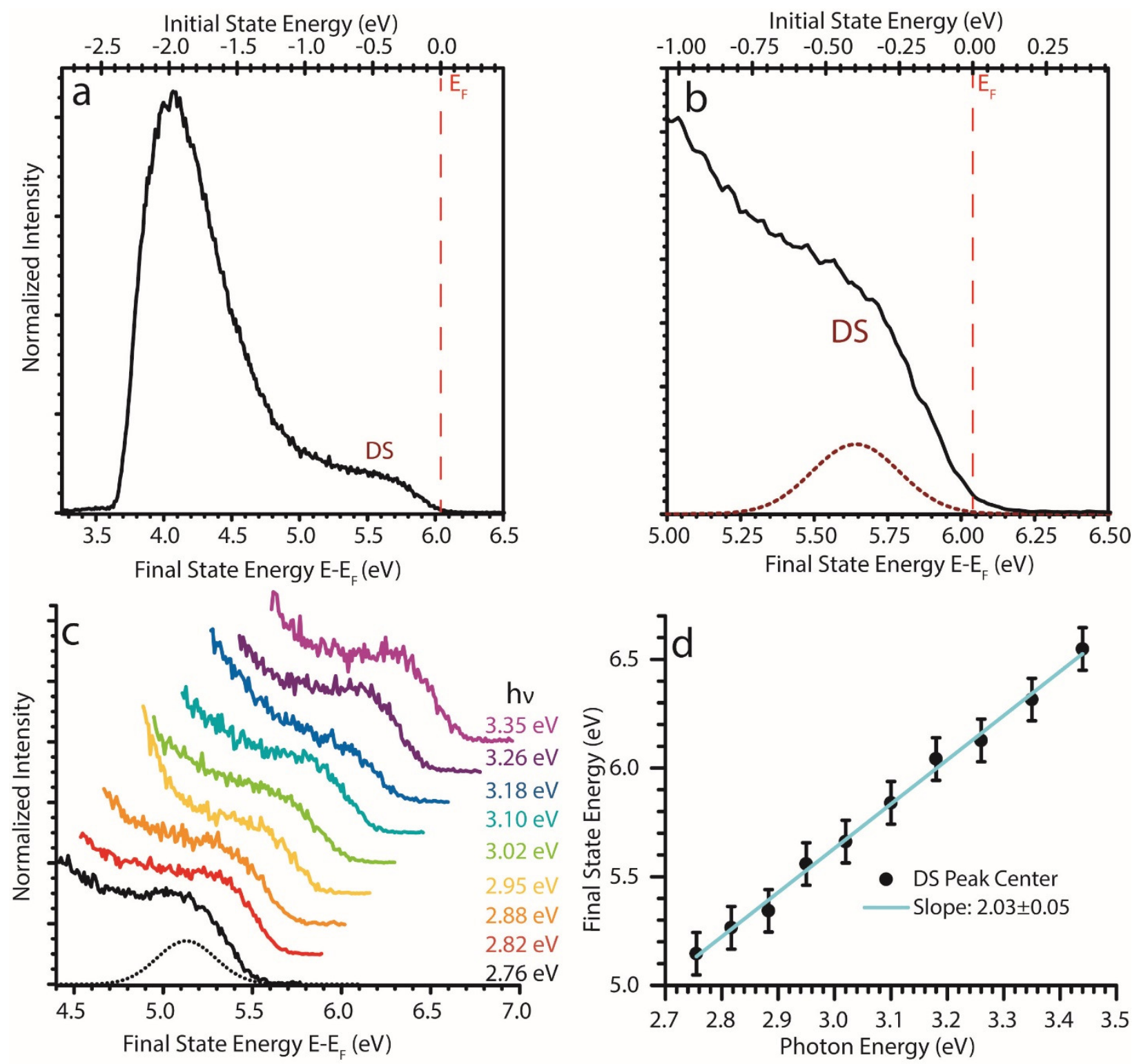

Since 2 PPE simultaneously probes both the occupied and unoccupied levels, the origin of a spectroscopic feature can be identified by measuring the final state energy as a function of photon energy. To this end, the excitation energy was varied over a range of $600 \mathrm{meV}$. The DS 
feature is observed at all energies (Fig. 3c), shifting to higher final state energy with increased photon energy. The mild variation in intensity is likely caused by slight spot to spot variation of the inhomogeneous polycrystalline surface, as well as wavelength-dependent changes in the laser spot sizes. When plotting $E_{\text {final }}$ vs. $E_{\text {photon }}($ Fig. 3d), we observe a linear dependence of the fitted peak center with a slope of 2.03(5). This indicates unambiguously that DS arises by two-photoninduced photoemission from an occupied level. DS was observed on all samples independent of excitation intensities. The high final state energy and linear dependence of $2 h v$ of the observed state combined with the insufficient energies for excitation from the valence band

maximum, $2 h v<E_{g}+\Phi$, further confirm the identification of an occupied gap state located near $E_{\mathrm{F}}$. The binding energy of DS, estimated from the center of the fitted peak, is $-0.37(3) \mathrm{eV}$ below $E_{F}$. 2PPE of such conductive $\mathrm{ZnO}$ films reveals therefore a density of states near $E_{F}$ that is not observed in UPS, due to its band gap selectivity and difference in photoemission cross-section. This state is a likely a contributor to the $n$-character of these films.

The surface-sensitive nature of 2PPE indicates that the species responsible for DS is present in significant quantities in the near-surface region of $\mathrm{ZnO}$. This suggests that the DS density of states is amenable to manipulation by surface structure modification. We use this as a means to develop next insight into the origin of the DS state and to understand the influence of molecular surface functionalization.

\section{(c) Gap State Modulation by $\mathrm{C}_{60}$ Adsorption}

The donor nature of DS can be further confirmed by adsorption of a strong molecular acceptor such as $\mathrm{C}_{60}$. Such molecules have been suggested previously to be able to accept electrons from shallow donor states in $\mathrm{ZnO}$, resulting in the formation of a hybrid interface state 
observed already at sub-monolayer coverages. ${ }^{5,24,25,27}$ Therefore, incremental coverages of $\mathrm{C}_{60}$, from sub-monolayer to bulk, were deposited and monitored by both UPS and 2PPE. Upon deposition of 1 MLE, the workfunction increases by $0.6(1) \mathrm{eV}$, further increasing by a total of 0.9(1) eV over 6 MLE. Such a workfunction increase indicates charge transfer from $\mathrm{ZnO}$ to $\mathrm{C}_{60}$ (Figure 4a), as reported elsewhere. ${ }^{5,24,25}$ In UPS, the spectra are dominated by the emergence of $\mathrm{C}_{60}$ valence band features (Figure $4 \mathrm{~b}$ ). Careful inspection of the energy gap region by $2 \mathrm{PPE}$ demonstrates that already at $4 \AA$ coverage (0.5 MLE), DS is completely quenched (Figure 4c). The hybrid interface state, observed in UPS and explicitly discussed previously, ${ }^{5,24,25}$ is not however clearly identifiable by 2PPE, likely due to differences in photoionization cross-section and a higher background of inelastically scattered photoelectrons.

Figure 4: (a) UP-spectra at the secondary electron cut-off for $\mathrm{ZnO}$, showing workfunction change with varying coverages of $\mathrm{C}_{60}$. (b) Overview UP-spectra as a function of $\mathrm{C}_{60}$ coverage. (c) 2PPE spectra at a photon energy of $3.04 \mathrm{eV}$ for bare $\mathrm{ZnO}, 0.5 \mathrm{MLE}(4 \AA)$, and $1 \mathrm{MLE}(8 \AA$ ) $\mathrm{C}_{60}$, offset for clarity.
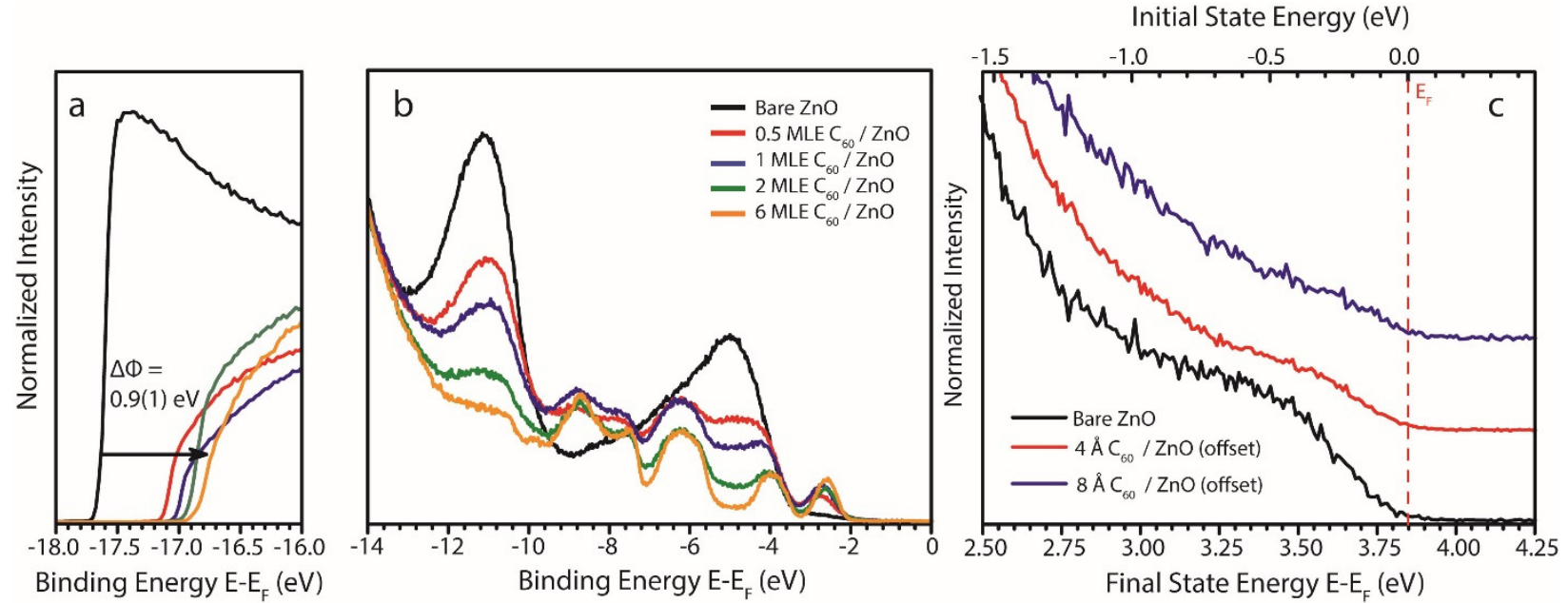
The binding energy of DS and its behavior upon $\mathrm{C}_{60}$ deposition is consistent with the view that DS is a shallow donor defect that undergoes charge transfer to $\mathrm{C}_{60}$. Consequently, $\mathrm{C}_{60}$ adsorption removes the occupied gap state density of DS. As demonstrated by Schulz et al. ${ }^{24}$, such interfacial interactions between $\mathrm{ZnO}$ and organic layers can improve charge extraction or act as centers for trapping and exciton recombination. The observation of DS by 2PPE and its quenching by $\mathrm{C}_{60}$ suggests therefore strongly that this shallow defect level corresponds to a nearsurface derived defect state and is closely involved in establishing the interfacial electronic structure and energy level alignment across this hybrid interface. Moreover, our results show clearly that band gap selective 2PPE is useful for directly detecting defects and surface derived gap states as well as the effects of interface formation. This is important for furthering the understanding of hybrid organic / inorganic semiconductor interfaces. 5,10,24-26 $^{2}$

\section{(d) Gap State Modulation by Annealing}

The importance of the observed DS for the electronic structure of the near-surface region can be further confirmed by photoemission spectroscopy of $\mathrm{ZnO}$ thin film surfaces whose electronic structure has been manipulated in a controlled fashion. In particular, elevated temperatures may alter the concentration of kinetically trapped point defects in the near-surface region of the $\mathrm{ZnO}$ film. Commonly used annealing protocols for single crystalline $\mathrm{ZnO}$ surfaces at temperatures in excess of $500{ }^{\circ} \mathrm{C}$ are not appropriate for the peALD $\mathrm{ZnO}$ thin films grown on IZO coated glass, since they lead to excessive interdiffusion of In from the substrate. We therefore restricted annealing temperatures to $130{ }^{\circ} \mathrm{C}$, where no evidence of indium diffusion was detected by XPS. After $1 \mathrm{~h}$ at $130^{\circ} \mathrm{C}$, noticeable changes in both $2 \mathrm{PPE}$ and UP spectra were observed, as shown in Figure 5. Remarkably, the spectral feature of DS is completely quenched in the 2PPE spectra (Figure 5a). In the UP spectra (Figures 5b-d), we observe a work function increase of $0.5(1) \mathrm{eV}$ 
(Figure 5b), while the valence band up-shifts by $0.2(1) \mathrm{eV}$ only (Figure 5c). This indicates the development of a surface dipole of $\Delta_{S D}=0.3(1) \mathrm{eV}$, accompanied by mild band bending of $\Delta_{B B}=+0.2(1) \mathrm{eV}$. This band bending is also confirmed by O $1 \mathrm{~s}$ and $\mathrm{Zn} 2 \mathrm{p}$ XPS (Figure 6) and is also consistent with the model put forth by Schlesinger et al. in the limit of high carrier densities. ${ }^{36}$ A summary of the energetics is shown in Figure 5d, and suggests together with the 2PPE spectra that annealing causes either a removal of dopants or an emptying of doping states in the near-surface region. We note that these results are not consistent with identifying DS as derived from adsorption of adventitious species such as $\mathrm{H}_{2} \mathrm{O}$ that may be still present at low concentration in UHV, since no reemergence of DS is observed even $72 \mathrm{~h}$ after annealing, and the workfunction remains fixed at $4.2(1) \mathrm{eV}$. In summary, the observed changes to the electronic structure and the band bending strongly suggest that DS resides indeed near the $\mathrm{ZnO}$ surface, consistent with its quenching upon adsorption of $\mathrm{C}_{60}$ discussed in the previous section.

Figure 5: (a) Normalized 2PPE spectra of annealed vs. unannealed $\mathrm{ZnO}$. (b) UPS with workfunction change and (c) VB shift upon annealing. (d) Schematic representation of changes to surface electronic structure upon annealing. 
Initial State Energy (eV)
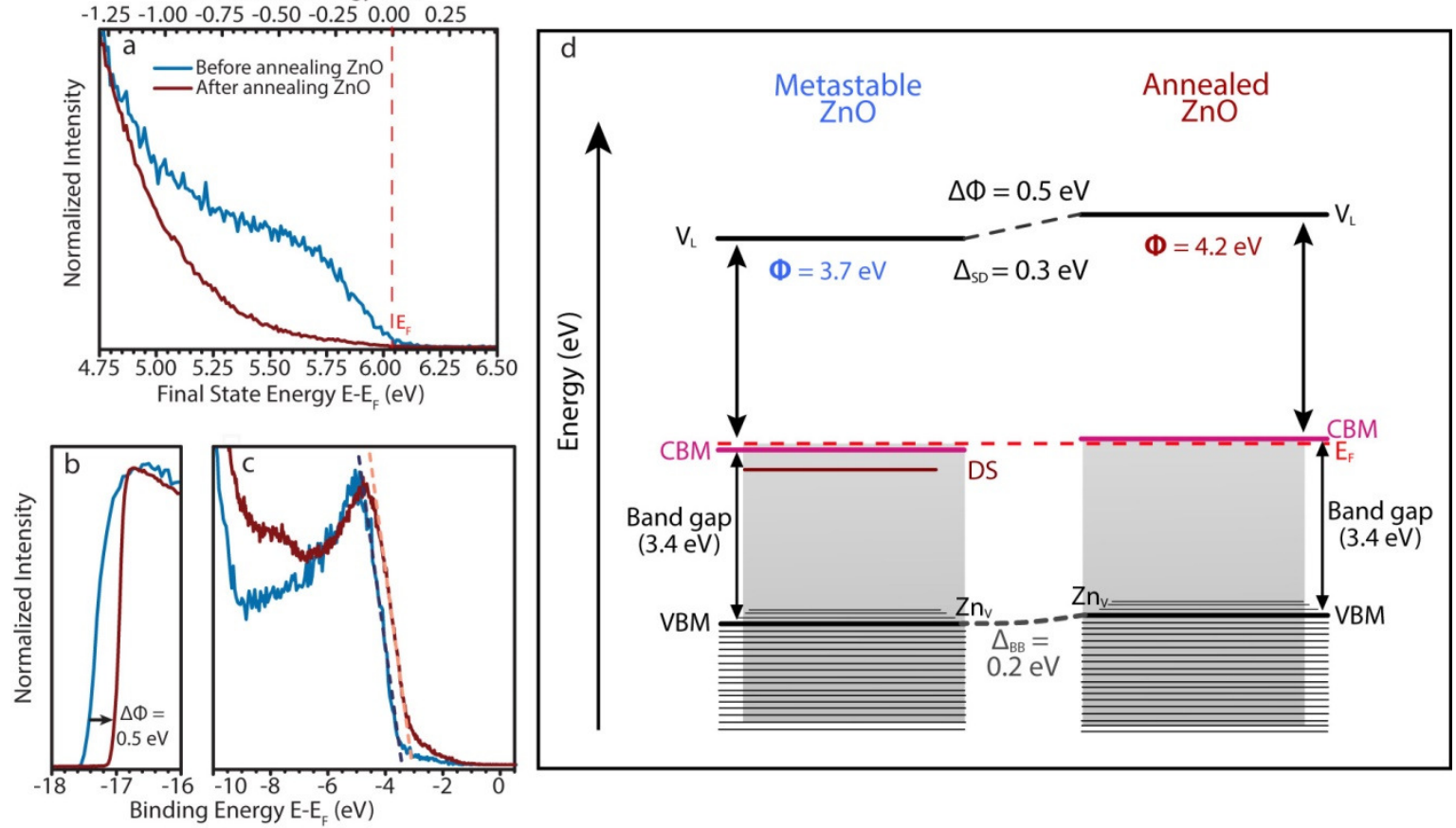

Figure 6: X-ray photoemission spectra for unsputtered and unannealed peALD ZnO vs. sputtered and annealed peALD ZnO. Spectral shifts are indicated for (a) $\mathrm{Zn} 2 \mathrm{p}_{3 / 2}$ and (b) O $1 \mathrm{~s}$.
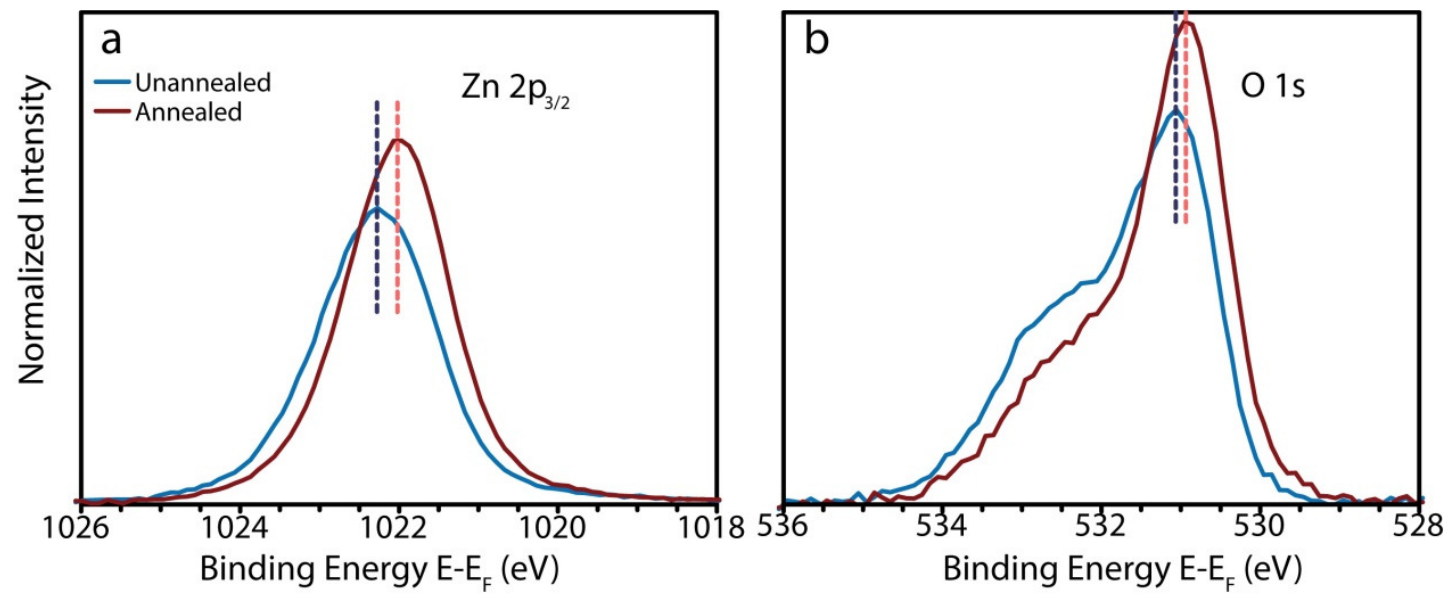

(e) Identification of the Gap State: Comparison with Theory

All experimental data suggest that DS is likely a donor-type defect state located near the $\mathrm{ZnO}$ film surface. Donor states in bulk $\mathrm{ZnO}$ are usually associated with interstitial $\mathrm{Zn}\left(\mathrm{Zn}_{\mathrm{i}}\right), \mathrm{O}$ 
vacancies $\left(\mathrm{O}_{\mathrm{v}}\right)$, or hydrogen impurities, the most common native or impurity donor defects in $\mathrm{ZnO}$. We briefly consider each of these possible origins of DS in turn in order to suggest an assignment of this gap state.

It has been well established that $\mathrm{Zn}_{\mathrm{i}}$ are shallow donors, within some meV of the conduction band minimum even in the near-surface region. ${ }^{9,10,22,23}$ Surface $\mathrm{Zn}_{\mathrm{i}}$, investigated for the $\mathrm{ZnO}$ (002) Zn-terminated surface, have also been shown to have density of states just at or below the conduction band minimum. ${ }^{10}$ The DS peak observed in the 2PPE spectra has a binding energy (peak center) of $E_{B}=-0.37(3) \mathrm{eV}$ (peak onset at $0 \mathrm{eV}$ ) and is thus too deep to be readily consistent with $\mathrm{Zn}_{\mathrm{i}}$. Moreover, the calculated formation energy of $\mathrm{Zn}_{\mathrm{i}}$ is rather high, at least for bulk $\mathrm{ZnO}$, substantially lowering the expected concentration of $\mathrm{Zn}_{\mathrm{i}}$ in the $\mathrm{ZnO}$ films. ${ }^{9,23}$ Even the formation of complexes between $\mathrm{Zn}_{\mathrm{i}}$ and $\mathrm{O}_{\mathrm{v}}$, suggested to stabilize $\mathrm{Zn}_{\mathrm{i}}$ and drastically increasing their concentration, ${ }^{11,23}$ cannot explain the nature of DS. The electronic structure of such defect complexes has been investigated in detail computationally in bulk $\mathrm{ZnO},{ }^{11,23}$ pushing $\mathrm{Zn}_{\mathrm{i}}$-derived levels even closer toward the conduction band minimum. The origin of DS must be therefore sought elsewhere.

Doping by hydrogen has been widely discussed as a possible source for the unintentional $n$ type nature of bulk $\mathrm{ZnO}$ and its surfaces, since $\mathrm{H}_{2}$ is present in some amount under most experimental conditions, including ultrahigh vacuum, ${ }^{37}$ and can be cracked to atomic hydrogen generated $e . g$. by hot filaments inside a vacuum chamber. Controlled surface adsorption of $\mathrm{H}$ on single crystalline $\mathrm{ZnO}$ showed that a partially occupied state emerges in the UP-spectra near $E_{F}$ at a binding energy of $\sim 0.1 \mathrm{eV}$, giving the $\mathrm{ZnO}$ surface a metallic character. ${ }^{8,30,31,38}$ The DS observed by 2 PPE is not observable by UPS, exhibits a much larger binding energy, and as discussed above, exhibits no time dependent spectral changes that would be consistent with 
adventitious $\mathrm{H}$. Electronic states arising from surface-adsorbed $\mathrm{H}$ atoms are thus also unlikely as the origin of DS.

In bulk $\mathrm{ZnO}$, oxygen vacancies have low formation energies under most conditions and are generally acknowledged as the most abundant donor defect in $\mathrm{ZnO} .{ }^{9,39} \mathrm{In}$ their neutral form, $\mathrm{O}_{\mathrm{v}}{ }^{0}$, oxygen vacancies in bulk $\mathrm{ZnO}$ are deep donor states situated close to the valence band maximum, in obvious conflict with the observed binding energy of $-0.37(3) \mathrm{eV}$ for DS..$^{9,23,40}$ More relevant might be however energy levels introduced by $\mathrm{O}_{\mathrm{v}}$ located near the $\mathrm{ZnO}$ surface. This situation, including the interplay with other point defects and $\mathrm{OH}$ adsorbates, has been recently considered in detail. ${ }^{10}$ Note that surface hydroxyls must be included since they are indeed commonly observed on most $\mathrm{ZnO}$ surfaces even after $\mathrm{Ar}^{+}$sputter treatment, as is evident also from the high binding energy shoulder in the O 1s XPS data (Figure 6b). ${ }^{5,10,39}$

On the polar $\mathrm{Zn}$-terminated (002) $\mathrm{ZnO}$ surface, oxygen vacancies at the surface, $\mathrm{O}_{V}^{S}$, cause strong relaxation of nearest neighbor zinc atoms and $\mathrm{Zn}-\mathrm{Zn}$ bond formation, ${ }^{10}$ as illustrated in Figure 7. This creates two shallow donor states at $-0.08 \mathrm{eV}$ and at $-0.35 \mathrm{eV}$ below the conduction band minimum. These states are much shallower than the reported values for bulk $\mathrm{O}_{\mathrm{v}}{ }^{10}{ }^{10}$ with the deeper of the two states in perhaps somewhat fortuitous agreement with the experimentally detected DS at $-0.37(3) \mathrm{eV}$. Previous calculations also confirm that defect bands for surface donor defects indeed tend to be closer to the CBM than in the bulk crystal. ${ }^{24,41}$ The calculated formation energy of $\mathrm{O}_{V}^{S}$ is however higher $(4.04 \mathrm{eV})$ than $\mathrm{O}_{\mathrm{V}}$ in bulk $\mathrm{ZnO}(3.72 \mathrm{eV}) .{ }^{9,10}$ This makes such defects less likely to occur naturally in high concentrations. The widely used external surface cleaning by $\mathrm{Ar}^{+}$bombardment is however well known to preferentially sputter $\mathrm{O}$ vs. $\mathrm{Zn}$ atoms, ${ }^{24}$ thus leading to increased densities of $\mathrm{O}_{V}^{S}$ despite the high formation energy needed under equilibrium conditions. ${ }^{10}$ Considering the metastable nature of DS, as established 
by annealing of the $\mathrm{ZnO}$ film, and its donor character, the assignment of $\mathrm{DS}$ as an $\mathrm{O}_{V}^{S}$ type defect offers thus a likely explanation for the observed gap state. We emphasize that such defects may be created by a broad variety of commonly used surface treatment protocols and may indeed be desirable since they convey a high degree of near-surface doping.

Thermodynamically more favorable than $\mathrm{O}_{\mathrm{v}}$, is the formation of $\mathrm{Zn}_{\mathrm{v}}$ at the surface, a deep acceptor defect common in $\mathrm{ZnO}{ }^{9}$ In the presence of $\mathrm{O}_{V}^{S}$, such $\mathrm{Zn}_{\mathrm{v}}$ sites prevent the formation of a metalized $\mathrm{ZnO}$ surface state (no $\mathrm{Zn}$ dimers), in good agreement with our experimental results after annealing. Computational studies have shown instead that $\mathrm{O}_{\mathrm{v}}-\mathrm{Zn}_{\mathrm{v}}$ donor-acceptor pairs are formed, with some of the $\mathrm{OH}$ molecules filling the neighboring $\mathrm{O}_{V}^{S}$ (Figure 7). The proposed presence of $\mathrm{Zn}_{\mathrm{v}}$ is in good agreement with the observation of a large tail density of states near the VBM in UPS (Figure 2c) and an in-depth analysis of O 1s XPS band. ${ }^{9,10}$

The two limiting cases, the metastable surface with $\mathrm{OH}$ and $\mathrm{O}_{V}^{S}$ and the surface with $\mathrm{OH}, \mathrm{Zn}_{\mathrm{v}}$ and $\mathrm{O}_{V}^{S}$, provide thus a basis for interpretation of DS as near-surface $\mathrm{O}_{V}^{S}$ states. We speculate that the mild sputtering conditions used during surface preparation are insufficient to form a large abundance of $\mathrm{Zn}-\mathrm{Zn}$ dimers that would otherwise metallize the surface and create a DOS at $-0.08 \mathrm{eV} .{ }^{10}$ Instead, the treatment introduces some $\mathrm{O}_{V}^{S}$ to form a metastable surface, modified by annealing, and supporting a shallow donor state quenched by charge transfer to $\mathrm{C}_{60}$. During annealing, the surface approaches a more stable surface composition $\left(\mathrm{OH}, \mathrm{Zn}_{\mathrm{v}}, \mathrm{O}_{V}^{S}\right)$, also consistent with the increased workfunction ${ }^{10}$ and the appearance of a new band at approximately $-8 \mathrm{eV}$ binding energy in UPS, suggested to arise from surface-localized $\mathrm{O} 2 \mathrm{p} \otimes \mathrm{Zn} 4 \mathrm{sp}$ hybridized states. $^{29,42}$ We note that the non-polar (100) surface, likewise present in the $\mathrm{ZnO}$ films investigated here, also supports $\mathrm{O}_{V}^{S}$ which are expected to occur at somewhat different energies. 
Figure 7: (left) Energy level diagram for the peALD $\mathrm{ZnO}$ film prior to (left) and after annealing (right), including calculated native point defect levels. Cartoon illustrating the conversion of a metastable $\mathrm{ZnO}$ surface with $\mathrm{O}_{V}^{S}$ (yellow circle) and $\mathrm{OH}$ (upper right panel, Model 3 in Ref [10]) to a surface with $\mathrm{Zn}_{\mathrm{v}}$ (blue circle), filled $\mathrm{O}_{V}^{S}$, and $\mathrm{OH}$ after annealing (lower right panel, Model 4 in $\operatorname{Ref}[10])$.
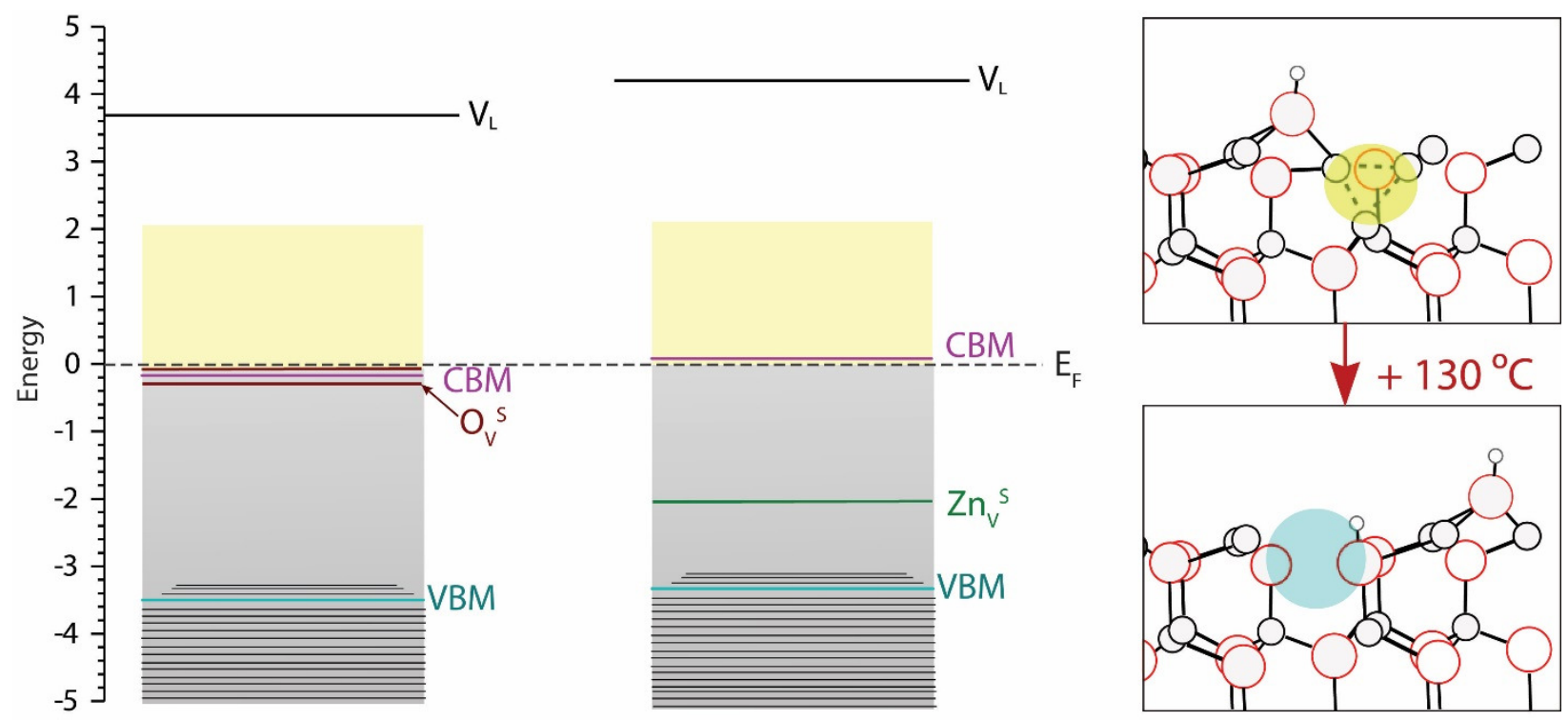

\section{Conclusions}

Using two-photon photoemission we have obtained direct spectroscopic evidence for the presence of a shallow donor defect in the near-surface region of conductive $\mathrm{ZnO}$ films. Based on the electronic structure of model $\mathrm{ZnO}$ surfaces with surface-confined point defects, we identify this density of states as arising from metastable $\mathrm{O}_{\mathrm{v}}$ located at the surface. Further support for this interpretation stems from the exquisite sensitivity of this density of states to the specific surface treatments. We have shown that the defect density is altered dramatically by mild annealing, which facilitates a transition from a metastable surface with shallow donors $\left(\mathrm{O}_{V}^{S}\right)$ to a surface that is otherwise dominated by deep acceptors $\left(\mathrm{Zn}_{\mathrm{V}}\right)$, adsorbed $\mathrm{OH}$, and filled $\mathrm{O}_{V}^{S}$. This transition is 
indicated by a considerable increase in workfunction, mild band bending and an interface dipole, both of which suggest the removal of $n$-type defects within the band gap. The defect density is also quenched by the adsorption of sub-monolayer layers of $\mathrm{C}_{60}(0.5 \mathrm{MLE})$, indicating interfacial hybridization and charge transfer between a donor defect and the acceptor molecule.

Beyond the specific assignment of surface gap states in $\mathrm{ZnO}$, our results demonstrate that near-surface point defects can be distinguished with surface sensitive two-photon photoemission spectroscopy. Our study provides improved understanding and direct spectroscopic evidence for surface-confined defects in the prototypical transparent conductive oxide, $\mathrm{ZnO}$. For highly conductive interlayers and carrier harvesting layers, our results increase the ability to control the surface electronic structure through both the type and the density of specific defects. This control allows for the rational design of hybrid organic / inorganic interfaces with desired electronic properties, and is may be relevant to hybrid interfaces beyond $\mathrm{ZnO}$ such as other oxides or perovskites.

\section{Acknowledgments}

This work was supported as part of the Center for Interface Science: Solar Electric Materials (CISSEM), an Energy Frontier Research Center funded by the U.S. Department of Energy, Office of Science, Basic Energy Sciences under Award Number DE-SC0001084. 


\section{References}

(1) Ratcliff, E. L.; Zacher, B.; Armstrong, N. R. J. Phys. Chem. Lett. 2011, 2 (11), 13371350.

(2) White, M. S.; Olson, D. C.; Shaheen, S. E.; Kopidakis, N.; Ginley, D. S. Appl. Phys. Lett. 2006, 89 (14), 143517.

(3) Cheun, H.; Fuentes-Hernandez, C.; Zhou, Y.; Potscavage, W. J.; Kim, S.-J.; Shim, J.; Dindar, A.; Kippelen, B. J. Phys. Chem. C 2010, 114 (48), 20713-20718.

(4) Steim, R.; Kogler, F. R.; Brabec, C. J. J. Mater. Chem. 2010, 20 (13), 2499-2512.

(5) Winget, P.; Schirra, L. K.; Cornil, D.; Li, H.; Coropceanu, V.; Ndione, P. F.; Sigdel, A. K.; Ginley, D. S.; Berry, J. J.; Shim, J.; Kim, H.; Kippelen, B.; Brédas, J.-L.; Monti, O. L. A. Adv. Mater. 2014, 26 (27), 4711-4716.

(6) Xu, Y.; Hofmann, O. T.; Schlesinger, R.; Winkler, S.; Frisch, J.; Niederhausen, J.; Vollmer, A.; Blumstengel, S.; Henneberger, F.; Koch, N.; Rinke, P.; Scheffler, M. Phys. Rev. Lett. 2013, 111 (22), 226802.

(7) Verdenhalven, E.; Knorr, A.; Richter, M.; Bieniek, B.; Rinke, P. Phys. Rev. B 2014, 89 (23), 235314.

(8) Hofmann, O. T.; Deinert, J.-C.; Xu, Y.; Rinke, P.; Stahler, J.; Wolf, M.; Scheffler, M. J. Chem. Phys. 2013, 139 (17), 174701.

(9) Janotti, A.; Van de Walle, C. Phys. Rev. B 2007, 76, 165202-165222.

(10) Li, H.; Schirra, L. K.; Shim, J.; Cheun, H.; Kippelen, B.; Monti, O. L. A.; Bredas, J.-L. Chem. Mater. 2012, 24, 3044-3055.

(11) Kim, Y.-S.; Park, C. H. Phys. Rev. Lett. 2009, 102, 086403.

(12) Brillson, L. J.; Dong, Y.; Tuomisto, F.; Svensson, B. G.; Kuznetsov, A. Y.; Doutt, D.; Mosbacker, H. L.; Cantwell, G.; Zhang, J.; Song, J. J.; Fang, Z.-Q.; Look, D. C. Phys. Status Solidi C 2012, 9 (7), 1566-1569.

(13) Djurišić, A. B.; Choy, W. C. H.; Roy, V. a. L.; Leung, Y. H.; Kwong, C. Y.; Cheah, K. W.; Gundu Rao, T. K.; Chan, W. K.; Fei Lui, H.; Surya, C. Adv. Funct. Mater. 2004, 14 (9), 856-864.

(14) Evans, S. M.; Giles, N. C.; Halliburton, L. E.; Kappers, L. A. J. Appl. Phys. 2008, 103 (4), 043710.

(15) Halliburton, L. E.; Giles, N. C.; Garces, N. Y.; Luo, M.; Xu, C.; Bai, L.; Boatner, L. A. Appl. Phys. Lett. 2005, 87 (17), 172108.

(16) Vlasenko, L. S.; Watkins, G. D. Phys. Rev. B 2005, 71, 125210-125215.

(17) Frenzel, H.; v. Wenckstern, H.; Weber, A.; Schmidt, H.; Biehne, G.; Hochmuth, H.; Lorenz, M.; Grundmann, M. Phys. Rev. B 2007, 76 (3), 035214.

(18) Ellguth, M.; Schmidt, M.; Pickenhain, R.; Wenckstern, H. V.; Grundmann, M. Phys. Status Solidi B 2011, 248 (4), 941-949.

(19) Chicot, G.; Muret, P.; Santailler, J.-L.; Feuillet, G.; Pernot, J. J. Phys. Appl. Phys. 2014, 47 (46), 465103.

(20) Seebauer, E. G.; Kratzer, M. C. Mater. Sci. Eng. R Rep. 2006, 55 (3-6), 57-149.

(21) Janotti, A.; Van de Walle, C. G. Nat. Mater. 2007, 6 (1), 44-47.

(22) Oba, F.; Choi, M.; Togo, A.; Tanaka, I. Sci. Technol. Adv. Mater. 2011, 12 (3), 034302.

(23) Kim, M. S.; Kim, Y.-S.; Park, C. H. Curr. Appl. Phys. 2011, 11, 5288-5291.

(24) Schulz, P.; Kelly, L. L.; Winget, P.; Li, H.; Kim, H.; Ndione, P. F.; Sigdel, A. K.; Berry, J. J.; Graham, S.; Brédas, J.-L.; Kahn, A.; Monti, O. L. A. Adv. Funct. Mater. 2014, 24 (46), 7381-7389. 
(25) Gruenewald, M.; Schirra, L. K.; Winget, P.; Kozlik, M.; Ndione, P. F.; Sigdel, A. K.; Berry, J. J.; Forker, R.; Brédas, J.-L.; Fritz, T.; Monti, O. L. A. J. Phys. Chem. C 2015, 119 (9), 4865-4873.

(26) Racke, D. A.; Monti, O. L. A. Surf. Sci. 2014, 630, 136-143.

(27) Racke, D. A.; Kelly, L. L.; Kim, H.; Schulz, P.; Sigdel, A.; Berry, J. J.; Graham, S.; Nordlund, D.; Monti, O. L. A. J. Phys. Chem. Lett. 2015, 1935-1941.

(28) Deinert, J.-C.; Wegkamp, D.; Meyer, M.; Richter, C.; Wolf, M.; Stähler, J. Phys. Rev. Lett. 2014, 113 (5), 057602.

(29) Ozawa, K.; Sawada, K.; Shirotori, Y.; Edamoto, K. J. Phys. Condens. Matter 2005, 17 (8), 1271.

(30) Ozawa, K.; Mase, K. Phys. Rev. B 2010, 81, $205322-205327$.

(31) Ozawa, K.; Mase, K. Phys. Rev. B 2011, 83, 125406-125408.

(32) Kinoshita, I.; Anazawa, T.; Matsumoto, Y. Chem. Phys. Lett. 1996, 259 (3-4), 445-450.

(33) Winkelmann, A.; Lin, W.-C.; Chiang, C.-T.; Bisio, F.; Petek, H.; Kirschner, J. Phys. Rev. B 2009, 80 (15), 155128.

(34) Onda, K.; Li, B.; Zhao, J.; Jordan, K. D.; Yang, J.; Petek, H. Science 2005, 308 (5725), 1154-1158.

(35) Onda, K.; Li, B.; Petek, H. Phys. Rev. B 2004, 70 (4), 045415.

(36) Schlesinger, R.; Xu, Y.; Hofmann, O. T.; Winkler, S.; Frisch, J.; Niederhausen, J.; Vollmer, A.; Blumstengel, S.; Henneberger, F.; Rinke, P.; Scheffler, M.; Koch, N. Phys. Rev. B 2013, 87 (15), 155311.

(37) Bacher, J.-P.; Benvenuti, C.; Chiggiato, P.; Reinert, M.-P.; Sgobba, S.; Brass, A.-M. J. Vac. Sci. Technol. A 2003, 21 (1), 167-174.

(38) Ozawa, K.; Sato, T.; Oba, Y.; Edamoto, K. J. Phys. Chem. C 2007, 111, 4256-4263.

(39) Wöll, C. Prog. Surf. Sci. 2007, 82, 55-120.

(40) Janotti, A.; Walle, C. G. V. de. Appl. Phys. Lett. 2005, 87 (12), 122102.

(41) D’Amico, N. R.; Cantele, G.; Ninno, D. J. Phys. Chem. C 2012, 116 (40), 21391-21400.

(42) Ozawa, K.; Oba, Y.; Edamoto, K.; Higashiguchi, M.; Miura, Y.; Tanaka, K.; Shimada, K.; Namatame, H.; Taniguchi, M. Phys. Rev. B 2009, 79 (7), 075314. 